\title{
Plasmon-activated water is an effective agent in suppressing the progression of dental, metabolic, and neurodegenerative disorders
}

\author{
H.-M. Chang ${ }^{1 *}$, T.Y. Renn ${ }^{2}$, Y.C. Liu 3 , F.D. Mai ${ }^{3}$, L.Y. Chen ${ }^{4}$ \\ ${ }^{1}$ Department of Anatomy and Cell Biology, Taipei Medical University, Taipei, Taiwan \\ ${ }^{2}$ Graduate Institute of Medical Sciences, Taipei Medical University, Taipei, Taiwan \\ ${ }^{3}$ Department of Biochemistry and Molecular Cell Biology, Taipei Medical University, Taipei, Taiwan \\ ${ }^{4}$ Department of Anatomy, Chung Shan Medical University, Taichung, Taiwan \\ *e-mail: taiwanzoo@gmail.com
}

Key words: periodontitis, diabetes mellitus, Alzheimer's disease, Parkinson's disease, oxidative stress

Motivation and Aim: With the coming of the aging society, more and more people are suffered from chronic metabolic and neurodegenerative disorders in which oxidative stress and systemic inflammation may underlie the molecular mechanism of these deficiencies. During the past few decades, a variety of drugs have been developed to depress or counteract the progression of such dysfunction. However, none of these drugs can persistently maintain their function. Moreover, their modest benefits are often offset by severe side effects. With regard to this viewpoint, developing a natural agent with no cytotoxicity, high biocompatibility, and significant anti-oxidative and anti-inflammatory properties will be of great help in rescuing the metabolic and neuronal function disrupted by related sequelae.

Methods and Algorithms: By letting the deionized water (DIW) flow through supported gold nanoparticles (Au NPs) under resonant illumination, we have developed the plasmonactivated water (PAW) with small water clusters that are more active in various chemical and physical reactions [1]. Adult rats subjected to periodontitis, diabetes mellitus, sleep deprivation, Alzheimer's disease, and Parkinson's disease were daily drunk of PAW, and the potential effects of PAW on protecting the cell function were comprehensively assessed by oxidative status, morphological profiles, molecular signaling in biochemical reaction, bio-energetic level as well as the metabolic and cognitive expressions. Results: Significant improvement including decreased oxidative stress, depressed inflammatory responses, enhanced anti-oxidative enzymes expression, increased bioenergetic level, as well as successful recovery of metabolic and cognitive functions were all clearly detected in diseased animals daily drunk of PAW as compared to that of rats received DIW treatment along.

Conclusion: As PAW could effectively suppress the progression of numerous dental, metabolic and neurodegenerative disorders, daily drinking of PAW may serve as a valuable strategy to improve the cellular function with a healthier, cheaper, more natural, and more convenient way.

Acknowledgements: Supported by the Ministry of Science and Technology (MOST 1062314-B038-003).

\section{References}

1. Chen H.C. et al. (2014) Active and stable liquid water innovatively prepared using resonantly illuminated gold nanoparticles. ACS Nano. 8:2704-2713.

2. Chen H.C. et al. (2018) Plasmon-activated water effectively relieves hepatic oxidative damage resulted from chronic sleep deprivation. RSC Advances. 8:9618-9626. 